\title{
Efeito da Aplicação de Nitrogênio na Produção e Qualidade da Madeira e Carvão Vegetal de um Híbrido de Eucalyptus grandis $x$ Eucalyptus urophylla
}

\author{
Claudinéia Olímpia Assis ${ }^{1}$, Paulo Fernando Trugilho ${ }^{1}$, Selma Lopes Goulart², \\ Maíra Reis de Assis ${ }^{1}$, Maria Lúcia Bianchi ${ }^{3}$ \\ ${ }^{1}$ Departamento de Ciências Florestais, Universidade Federal de Lavras - UFLA, Lavras/MG, Brasil \\ ${ }^{2}$ Universidade Federal Rural da Amazônia - UFRA, Campus Parauapebas, Parauapebas/PA, Brasil \\ ${ }^{3}$ Departamento de Química, Universidade Federal de Lavras - UFLA, Lavras/MG, Brasil
}

\begin{abstract}
RESUMO
O trabalho teve como objetivo verificar o efeito de diferentes doses de nitrogênio na produção de matéria seca e na qualidade da madeira e carvão vegetal de um clone híbrido de Eucalyptus grandis x Eucalyptus urophylla, aos 6 anos de idade. Foram analisadas 40 árvores, sendo estudados 5 tratamentos em 4 blocos e amostradas 2 árvores por tratamento dentro de cada bloco. Os tratamentos consistiram da aplicação de $0 \mathrm{~kg}, 60 \mathrm{~kg}, 120 \mathrm{~kg}$ e $240 \mathrm{~kg} \mathrm{ha}^{-1}$ de nitrogênio $(\mathrm{N})$, como sulfato de amônio, com o objetivo de avaliar se a resposta ao sulfato de amônio era exclusivamente devida ao N. Foi aplicado ainda um tratamento adicional, com aplicação de $120 \mathrm{~kg}$. $\mathrm{ha}^{-1}$ de $\mathrm{N}$ como nitrato de amônio, totalizando 5 tratamentos. Foram avaliadas as características de crescimento da árvore, densidade básica, estimativa de massa seca, composição química e energética da madeira e as características físicas, químicas e energéticas do carvão. Observou-se que a produção de massa seca tendeu a aumentar com a aplicação da dosagem de nitrogênio. $\mathrm{O}$ efeito da dosagem de nitrogênio não influenciou a densidade básica e as características energéticas do carvão vegetal, porém os teores de nitrogênio elementar, extrativos totais e lignina na madeira apresentaram aumento com a nutrição aplicada. A nutrição utilizada não influenciou as características químicas e energéticas da madeira e do carvão vegetal.
\end{abstract}

Palavras-chave: nutrição, clone, qualidade.

\section{Effect of Nitrogen Application on the Production and Quality of Wood and Charcoal from a Hybrid of Eucalyptus grandis $\mathrm{x}$ Eucalyptus urophylla}

\begin{abstract}
The present work aimed to verify the effect of different dosages of nitrogen on the production of dry material, and wood and charcoal quality made from a six years old hybrid clone of Eucalyptus grandis $\mathrm{x}$ Eucalyptus urophylla. Overall 40 trees were analyzed and five treatments in four blocks were studied, with two trees sampled per treatment within each block. Treatments consisted in the application of $0,60,120$ and $240 \mathrm{~kg} \cdot \mathrm{ha}^{-1}$ of nitrogen $(\mathrm{N})$ as ammonium sulfate in order to evaluate if the response to such compound was exclusively due to $\mathrm{N}$. An additional treatment was applied with $120 \mathrm{~kg} \cdot \mathrm{ha}^{-1}$ of $\mathrm{N}$ as ammonium nitrate, totalizing five treatments. The tree growth, basic density, dry mass estimate, chemical and energetic composition of wood were evaluated, as well as physical, chemical and energetic characteristics of charcoal. The production of dry mass tended to increase with the nitrogen dosage applied. The effect of nitrogen dosage did not
\end{abstract}


influence the basic density and energetic characteristics of charcoal, however the content of elemental nitrogen, total extractives and lignin of wood increased with nutrition. Such application did not influence the chemical and energetic characteristics of wood and charcoal.

Keywords: nutrition, clone, quality.

\section{INTRODUÇÃO}

A disponibilidade biológica do nitrogênio $(\mathrm{N})$ no solo, juntamente com outros nutrientes, como fósforo, enxofre e potássio, tem relação direta com a produtividade agrícola. $\mathrm{O} N$ encontra-se em abundância na atmosfera na forma do gás $\mathrm{N}_{2}$, constituindo cerca de $80 \%$ do seu volume total, nessa forma quimicamente inerte e não disponível para os seres vivos. As formas disponíveis ou "combinadas" de N para a nutrição dos seres vivos incluem as combinações amoniacais $\left(\mathrm{NH} 4^{+}\right)$, nítricas $\left(\mathrm{NO}^{3-}\right)$ ou orgânicas $\left(\mathrm{R}-\mathrm{NH}^{2}\right)$ que são metabolizadas visando à construção de biomassa (Taiz \& Zeiger, 2009).

A adubação nitrogenada é uma das práticas que mais têm sido empregadas para atingir o aumento da produtividade (MacDonald \& Hubert, 2002). Em ambientes terrestres, o nitrogênio é frequentemente limitante para o crescimento das plantas, portanto a adição de fertilizantes ricos em nitrogênio pode ter profundos efeitos sobre a fisiologia da árvore e o acúmulo de biomassa (Geßler et al., 2004; Cooke et al., 2005).

Segundo Smethurst et al. (2003), o eucalipto pode responder à nutrição nitrogenada aumentando sua área foliar, com consequente aumento de volume de tronco. Entretanto, poucos estudos têm sido conduzidos com as espécies florestais, especialmente em condições tropicais. Apesar de os trabalhos destacarem a importância do $\mathrm{N}$ para o crescimento das plantas, falta informação sobre as modificações sobre a qualidade do lenho produzido em resposta ao intenso desenvolvimento do vegetal. Modificações em níveis anatômicos e composicional têm sido frequentemente descritas em relação à fertilização com N-P-K, o stress de água e os estímulos mecânicos. Porém os efeitos da adubação sobre a qualidade da madeira são difíceis de predizer (Barbosa et al., 2014).

Ainda são poucos os estudos que relacionam diretamente a interferência dos atributos do solo sobre a qualidade de espécies florestais (Rigatto et al., 2005). Pode-se observar que existem informações contrastantes na literatura sobre a resposta da madeira de eucalipto à nutrição nitrogenada. Há, portanto, necessidade de novos estudos que avaliem o efeito da adubação nitrogenada tanto sobre o crescimento como sobre a composição química das árvores de eucalipto, visando determinar a dosagem adequada que possa ser aplicada pelas empresas produtoras dessa matéria-prima. Nesse contexto, este trabalho teve como objetivo avaliar o efeito do nitrogênio nas características de crescimento da árvore, qualidade da madeira e do carvão vegetal de um clone híbrido de Eucalyptus grandis $\mathrm{x}$ Eucalyptus urophylla aos 6 anos de idade, plantado para produção de carvão vegetal.

\section{MATERIAL E MÉTODOS}

\subsection{Coleta e preparo do material}

Foram analisadas 40 árvores aos 6 anos de idade de um clone híbrido de Eucalyptus grandis $\mathrm{x}$ Eucalyptus urophylla plantado em espaçamento $3 \mathrm{~m} \times 3 \mathrm{~m}$. Esse material proveio da empresa APERAM Bioenergia, situada no alto do Vale do Jequitinhonha, município de Itamarandiba, MG, 1751'26"S e 42 51'32"W, 1.097 m de altitude, precipitação média anual de $1.081 \mathrm{~mm}$ e temperatura média de $20,1^{\circ} \mathrm{C}$. Foram estudados 5 tratamentos em 4 blocos, nos quais foram amostradas 2 árvores por tratamento dentro de cada bloco. O experimento foi implantado em junho de 2005. Fontes, doses e épocas de aplicação encontram-se na Tabela $1 \mathrm{e}$ as dosagens de cada elemento químico estão apresentadas na Tabela 2.

\subsection{Características de crescimento da árvore}

Para as características de crescimento da árvore foram utilizados os dados de inventário contínuo na área experimental da empresa APERAM Bioenergia. A estimativa de massa seca (MS) foi determinada em função da densidade básica média (DBm) e o volume sem casca (VSC), conforme a Equação 1.

$M S=D B m \times V S C$ 


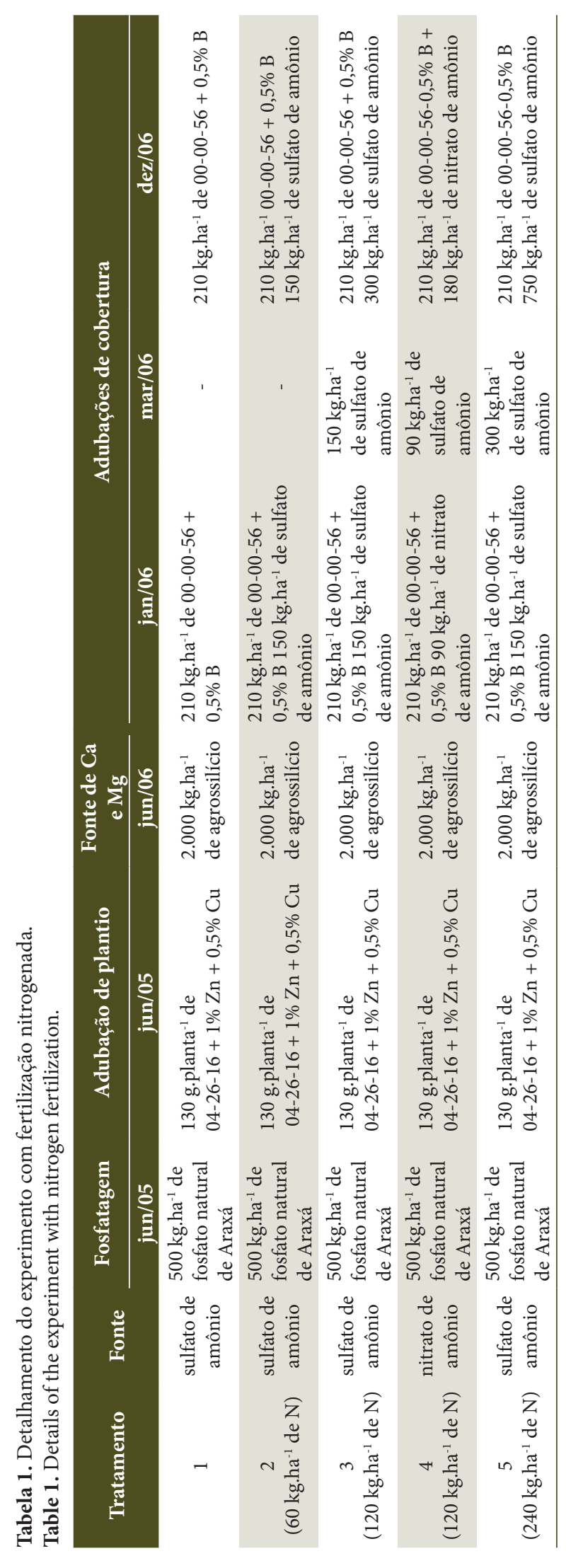


Tabela 2. Quantidade total de nutrientes aplicados nos diferentes tratamentos.

Table 2. Total amount of nutrients applied in the different treatments.

\begin{tabular}{|c|c|c|c|c|c|c|c|c|c|}
\hline \multirow{2}{*}{ Tratamento } & \multicolumn{9}{|c|}{ Dosagem dos nutrientes $\left(\mathrm{kg} \cdot \mathrm{ha}^{-1}\right)$} \\
\hline & $\mathrm{P}_{2} \mathrm{O}_{5}$ (total) & $\mathbf{N}$ & $\mathrm{K}_{2} \mathrm{O}$ & $\mathrm{Ca}$ & $\mathrm{Mg}$ & $\mathrm{Cu}$ & $\mathrm{Zn}$ & B & $\mathbf{S}$ \\
\hline 1 & 224,36 & 5,72 & 258,08 & 900,0 & 360,0 & 0,72 & 1,43 & 2,10 & 0,0 \\
\hline 2 & 224,36 & 125,72 & 258,08 & 900,0 & 360,0 & 0,72 & 1,43 & 2,10 & 72,0 \\
\hline 3 & 224,36 & 245,72 & 258,08 & 900,0 & 360,0 & 0,72 & 1,43 & 2,10 & 288,0 \\
\hline 4 & 224,36 & 244,52 & 258,08 & 900,0 & 360,0 & 0,72 & 1,43 & 2,10 & 0,0 \\
\hline 5 & 224,36 & 485,72 & 258,08 & 900,0 & 360,0 & 0,72 & 1,43 & 2,10 & 576,0 \\
\hline
\end{tabular}

\subsection{Determinação da densidade básica da madeira}

Para a determinação da densidade básica da madeira foi utilizado o procedimento descrito na norma NBR 11941 (ABNT, 2003). Foram retirados discos de $2,5 \mathrm{~cm}$ de espessura a $0 \%, 25 \%, 50 \%, 75 \%$ e $100 \%$ da altura comercial da árvore. Os discos foram divididos em quatro cunhas, passando pela medula, sendo utilizadas duas opostas. Determinou-se a densidade básica média da árvore $(\mathrm{DBm})$ como sendo a média aritmética dos pontos de amostragem longitudinal no tronco.

\subsection{Determinação química da madeira}

As amostras para a análise química da madeira foram retiradas das outras duas cunhas opostas, formando uma amostra composta por todos os pontos longitudinais. Esse material foi moído e classificado em peneiras sobrepostas de 40 mesh e 60 mesh, utilizando-se a fração retida na peneira de 60 mesh. Em seguida foi levado para a câmara de climatização com temperatura de $20^{\circ} \mathrm{C} \pm 3{ }^{\circ} \mathrm{C}$ e umidade relativa de $65 \% \pm 2 \%$, permanecendo nessa condição por aproximadamente 15 dias, para a homogeneização da umidade.

Foram determinados os teores de extrativos totais de acordo com as normas M3/89 e o teor de cinzas pela norma M11/77 (ABCTP, 1974). O teor de lignina insolúvel (Klason) foi obtido de acordo com a metodologia proposta por Gomide \& Demuner (1986) e o teor de lignina solúvel (Klason) em ácido sulfúrico foi determinado de acordo com a metodologia proposta por Goldschimid (1971). O teor de lignina total foi obtido pelo somatório dos teores de lignina solúvel e insolúvel.

\subsection{Determinação química elementar da madeira}

Para a composição química elementar foram utilizados aproximadamente $2 \mathrm{mg}$ de fração retida em peneira de 270 mesh. As análises foram realizadas em um analisador universal da marca Elementar, modelo Vario Micro Cube, para a determinação dos teores de nitrogênio, carbono, hidrogênio, enxofre e, por diferença, oxigênio presentes na amostra.

\subsection{Determinação do poder calorífico da madeira}

Para a determinação do poder calorífico superior da madeira utilizou-se a fração retida na peneira de 60 mesh. Inicialmente, esse material foi seco em estufa a uma temperatura de $103^{\circ} \mathrm{C} \pm 2{ }^{\circ} \mathrm{C}$. O poder calorífico superior foi determinado em um calorímetro digital, modelo IKA C-200, conforme a norma NBR 8633 (ABNT, 1984).

\subsection{Carbonização da madeira}

Para a carbonização da madeira, as amostras foram retiradas das cunhas opostas. Foram utilizadas aproximadamente $500 \mathrm{~g}$ de material previamente seco em estufa a temperatura de $103^{\circ} \mathrm{C} \pm 2^{\circ} \mathrm{C}$. A temperatura inicial foi de $100{ }^{\circ} \mathrm{C}$ e a temperatura final de $450{ }^{\circ} \mathrm{C}$, permanecendo o material a esta temperatura por um período de 30 minutos.

Em seguida foram avaliados os rendimentos gravimétricos em carvão vegetal, líquido pirolenhoso condensado e gases não condensáveis, a densidade relativa aparente do carvão e a análise química imediata, visando à determinação dos teores de materiais voláteis, cinzas e, por diferença, o teor de carbono fixo. O rendimento em carbono fixo foi calculado segundo a Equação 2: 
$R C F=\frac{R G C \times T C F}{100}$

em que RCF = Rendimento em Carbono Fixo (\%); RGC $=$ Rendimento Gravimétrico da Carbonização (\%); e TCF = Teor de Carbono Fixo (\%).

As amostras utilizadas para o poder calorífico superior do carvão vegetal foram as mesmas da carbonização, e a metodologia utilizada foi a mesma do poder calorífico superior da madeira. Os dados foram submetidos à análise de variância e, quando verificadas diferenças significativas, aplicou-se o teste de Tukey, em nível de 5\% de significância.

\section{RESULTADOS E DISCUSSÃO}

\subsection{Características de crescimento, densidade básica e estimativa de massa seca}

$\mathrm{Na}$ Tabela 3 encontram-se os valores médios e o teste de comparação múltipla para as características de crescimento, densidade básica e estimativa de massa seca. Observa-se pelos dados médios que houve variação entre os tratamentos para todas as características de crescimento, o que pode estar relacionado com as diferenças e as quantidades das dosagens de nitrogênio aplicadas em cada tratamento. Para a estimativa de massa seca, o tratamento 1 foi diferente dos demais, sendo um reflexo do menor volume e densidade básica média da madeira. Entretanto, a dosagem de nitrogênio aplicada não influenciou a densidade básica da madeira em nenhum dos tratamentos.

A dose estimada de $\mathrm{N}$ foi bem semelhante à utilizada nos tratamentos 3 e 4, diferenciando-se pela ausência do enxofre (S) para o tratamento 4. Deve-se levar em consideração que o tratamento 3 apresenta como diferencial uma dose elevada de enxofre (S) e que essa pode ter contribuído também para o melhor crescimento das plantas nesse tratamento. Pode-se observar também que o tratamento 1 assemelha-se ao tratamento 4, diferenciando-se apenas na dosagem de nitrogênio, que pode ser responsável pelas variações encontradas nos valores para esses tratamentos. Dessa forma, o tratamento 3 pode ser o mais indicado para se estabelecer uma dosagem adequada de $\mathrm{N}$ especificamente para esse material genético. Os resultados estão de acordo com Silva et al. (2003), que analisaram clones híbridos de Eucalyptus aos 36 meses de idade com aplicação de até $320 \mathrm{~kg} \cdot \mathrm{ha}^{-1}$ de N (30\% na forma de nitrato de amônio e $70 \%$ na forma de sulfato de amônio). Apesar da não aplicação de S, esses autores observaram um incremento linear, atingindo ganhos de até $44 \%$ em relação à testemunha.

Independentemente das fontes utilizadas, tanto nitrato de amônio como sulfato de amônio, e das diferenças das elevadas quantidades das dosagens aplicadas nos tratamentos, a nutrição nitrogenada não influenciou as características químicas e energéticas da madeira, a qual se manteve praticamente estável para todos os tratamentos. Araújo et al. (2003) verificaram que o fornecimento de $\mathrm{N}$ até $486 \mathrm{~kg} \cdot \mathrm{ha}^{-1}$ (primeira cobertura com ureia e segunda e terceira com sulfato de amônio) proporcionou ganho linear no crescimento de clones de Eucalyptus em espodossolos na região sul da Bahia. Em avaliação feita aos 24 meses de idade, esses mesmos autores relataram incremento de até 109\% no crescimento em relação à testemunha. Entretanto, eles não avaliaram o efeito simultâneo do S. No Brasil, a demanda de $\mathrm{N}$ pelo eucalipto na idade de 4,5 anos varia de $150 \mathrm{~kg}$ a $327 \mathrm{~kg} \cdot \mathrm{ha}^{-1}$ (Santana et al., 2008), com

Tabela 3. Teste de médias para as características de crescimento, densidade básica e estimativa de massa seca da madeira.

Table 3. Test of means for growth traits, basic density and estimate of dry mass of wood.

\begin{tabular}{cllllc}
\multirow{2}{*}{ Tratamento } & \multicolumn{5}{c}{ Características da madeira } \\
\cline { 2 - 6 } & DAP & VCC & VSC & DBm & MS \\
\hline 1 & $15,49 \mathrm{~b}$ & $209,10 \mathrm{~b}$ & $201,65 \mathrm{~b}$ & $0,471 \mathrm{a}$ & $95,11 \mathrm{~b}$ \\
\hline 2 & $16,29 \mathrm{a}$ & $241,58 \mathrm{a}$ & $234,25 \mathrm{a}$ & $0,485 \mathrm{a}$ & $113,56 \mathrm{a}$ \\
\hline 3 & $16,68 \mathrm{a}$ & $255,96 \mathrm{a}$ & $248,56 \mathrm{a}$ & $0,481 \mathrm{a}$ & $119,68 \mathrm{a}$ \\
4 & $16,20 \mathrm{ab}$ & $236,59 \mathrm{ab}$ & $229,40 \mathrm{ab}$ & $0,479 \mathrm{a}$ & $109,89 \mathrm{a}$ \\
\hline 5 & $16,42 \mathrm{a}$ & $246,47 \mathrm{a}$ & $238,71 \mathrm{a}$ & $0,479 \mathrm{a}$ & $114,39 \mathrm{a}$ \\
\hline
\end{tabular}

DAP: diâmetro à altura do peito $(\mathrm{cm})$; VCC: volume com casca $\left(\mathrm{m}^{3} \mathrm{ha}^{-1}\right)$; VSC: volume sem casca $\left(\mathrm{m}^{3} \mathrm{ha}^{-1}\right)$; DBm: densidade básica média $\left(\mathrm{g} \mathrm{cm}^{-3}\right)$; MS: massa seca $\left(\mathrm{t} \cdot \mathrm{ha}^{-1}\right)$. Médias seguidas pela mesma letra na coluna não diferem entre si a $5 \%$ de probabilidade pelo teste de Tukey. 
valores mais baixos em solos arenosos. Assim, respostas à aplicação de $\mathrm{N}$ podem ser esperadas em solos com teores mais baixos de matéria orgânica, geralmente arenosos, como os solos dos estudos de Silva et al. (2003) e Araújo et al. (2003) ou, ainda, em locais nos quais as condições climáticas não favorecem a rápida decomposição da matéria orgânica (Jesus et al., 2012).

\subsection{Características químicas e energéticas da madeira}

$\mathrm{Na}$ Tabela 4 encontram-se os valores médios e o teste de comparação múltipla para as características químicas e energéticas da madeira. Verifica-se que as dosagens de nitrogênio aplicadas nos tratamentos, tanto de sulfato de amônio como de nitrato de amônio, não apresentaram diferenças significativas para todas as variáveis analisadas, a 5\% de probabilidade. Portanto, o efeito de tratamento não afetou significativamente nenhuma dessas características da madeira. Independentemente das fontes utilizadas e das elevadas diferenças das dosagens aplicadas nos tratamentos, a nutrição não influenciou as características químicas e energéticas da madeira.

Os valores médios estão de acordo com os resultados encontrados por Oliveira et al. (2012), que avaliaram os parâmetros de qualidade da madeira de clones de Eucalyptus pellita aos 5 anos de idade e as propriedades físicas e químicas do carvão vegetal, e aos encontrados por Neves et al. (2011), que avaliaram a qualidade da madeira e do carvão vegetal de três clones de Eucalyptus plantados em diferentes locais, exceto para os teores de extrativos totais, que foram inferiores aos do presente trabalho. Esses valores encontrados podem estar associados à nutrição aplicada, especialmente com relação ao tratamento 3 .
Observa-se que os valores médios estão de acordo com os citados por Neves et al. (2011), Reis et al. (2012) e Protásio et al. (2011), que avaliaram as características químicas da madeira e do carvão vegetal de clones de Eucalyptus, exceto para os teores de nitrogênio, que foram superiores aos normalmente encontrados na literatura. Esse resultado deve estar relacionado à nutrição nitrogenada utilizada no experimento. O tratamento 3 foi o que apresentou uma ligeira redução nos valores para o nitrogênio. Para o uso energético, é desejável que a biomassa apresente menores teores de nitrogênio na sua composição. A presença de nitrogênio na composição da madeira resulta na formação de óxidos de nitrogênio após a combustão. Sendo assim, são desejáveis diminutas quantidades desse componente elementar na combustão e no processo de carbonização da madeira (Bilgen \& Kaygusuz, 2008; Kumar et al., 2010). Segundo Huang et al. (2009), o teor de nitrogênio não apresenta relação positiva com o poder calorífico superior da biomassa.

\subsection{Avaliação dos rendimentos gravimétricos, química, energética e da densidade relativa aparente do carvão vegetal}

Na Tabela 5 estão os valores médios e o teste de comparação múltipla para os rendimentos gravimétricos da carbonização, os teores de materiais voláteis, cinzas, carbono fixo, poder calorífico superior e densidade relativa aparente do carvão vegetal. Observa-se que somente para a característica rendimento em líquido pirolenhoso o efeito da nutrição nitrogenada foi significativo. $\mathrm{O}$ tratamento 4 apresentou um valor mais elevado em relação ao tratamento 1 , o que pode estar relacionado às dosagens diferentes nos dois tratamentos, sendo que o tratamento 4 apresentou

Tabela 4. Teste de médias para as variáveis teor de extrativos totais, teor de lignina total, teor de cinzas, poder calorífico superior, nitrogênio, carbono, hidrogênio e oxigênio da madeira.

Table 4. Test of means for the variables content of total extractives, total lignin, ashes, superior calorific power, nitrogen, carbon, hydrogen and oxygen of wood.

\begin{tabular}{|c|c|c|c|c|c|c|c|c|}
\hline \multirow{2}{*}{ Tratamento } & \multicolumn{8}{|c|}{ Características da madeira } \\
\hline & TET & TLT & $\mathrm{TCz}$ & PCS & $\mathbf{N}$ & C & $\mathbf{O}$ & $\mathbf{H}$ \\
\hline 1 & $9,56 \mathrm{a}$ & $32,93 \mathrm{a}$ & $0,29 \mathrm{a}$ & $4642 \mathrm{a}$ & $0,79 \mathrm{a}$ & $46,90 \mathrm{a}$ & $6,15 \mathrm{a}$ & $46,16 \mathrm{a}$ \\
\hline 2 & $10,12 \mathrm{a}$ & $32,61 \mathrm{a}$ & $0,29 \mathrm{a}$ & $4690 \mathrm{a}$ & $0,82 \mathrm{a}$ & $46,58 \mathrm{a}$ & $6,05 \mathrm{a}$ & $46,55 \mathrm{a}$ \\
\hline 3 & $10,60 \mathrm{a}$ & 34,18 a & $0,34 \mathrm{a}$ & $4579 \mathrm{a}$ & $0,71 \mathrm{a}$ & 47,16 a & $6,36 \mathrm{a}$ & $45,77 \mathrm{a}$ \\
\hline 4 & $10,02 \mathrm{a}$ & $33,50 \mathrm{a}$ & $0,29 \mathrm{a}$ & $4681 \mathrm{a}$ & $0,80 \mathrm{a}$ & $47,08 \mathrm{a}$ & 6,11 a & $46,01 \mathrm{a}$ \\
\hline 5 & $10,38 \mathrm{a}$ & $32,78 \mathrm{a}$ & $0,28 \mathrm{a}$ & $4692 \mathrm{a}$ & $0,78 \mathrm{a}$ & 49,37 a & $5,71 \mathrm{a}$ & $44,14 \mathrm{a}$ \\
\hline
\end{tabular}


Tabela 5. Valores médios e teste de comparação múltipla para os rendimentos gravimétricos da carbonização, química imediata, física e energética do carvão vegetal.

Table 5. Test of means for the gravimetric yields of carbonization, immediate chemistry, physics and energetics of charcoal.

\begin{tabular}{ccccccccc}
\multirow{2}{*}{ Tratamento Características do carvão vegetal } \\
\cline { 2 - 9 } & RGC & RLP & RCF & TMV & TCz & TCF & PCSc & DRA \\
\hline 1 & $34,67 \mathrm{a}$ & $44,97 \mathrm{~b}$ & $24,93 \mathrm{a}$ & $27,85 \mathrm{a}$ & $0,28 \mathrm{a}$ & $71,92 \mathrm{a}$ & $7375 \mathrm{a}$ & $0,385 \mathrm{a}$ \\
2 & $33,97 \mathrm{a}$ & $48,47 \mathrm{ab}$ & $24,83 \mathrm{a}$ & $26,68 \mathrm{a}$ & $0,30 \mathrm{a}$ & $73,11 \mathrm{a}$ & $7435 \mathrm{a}$ & $0,385 \mathrm{a}$ \\
\hline 3 & $33,97 \mathrm{a}$ & $48,07 \mathrm{ab}$ & $25,25 \mathrm{a}$ & $25,44 \mathrm{a}$ & $0,37 \mathrm{a}$ & $74,35 \mathrm{a}$ & $7466 \mathrm{a}$ & $0,365 \mathrm{a}$ \\
\hline 5 & $34,24 \mathrm{a}$ & $50,00 \mathrm{a}$ & $25,00 \mathrm{a}$ & $26,92 \mathrm{a}$ & $0,38 \mathrm{a}$ & $73,03 \mathrm{a}$ & $7257 \mathrm{a}$ & $0,404 \mathrm{a}$ \\
\hline & $34,31 \mathrm{a}$ & $46,79 \mathrm{ab}$ & $24,95 \mathrm{a}$ & $26,95 \mathrm{a}$ & $0,31 \mathrm{a}$ & $72,74 \mathrm{a}$ & $7345 \mathrm{a}$ & $0,399 \mathrm{a}$ \\
\hline
\end{tabular}

RGC: rendimento gravimétrico do carvão (\%); RLP: rendimento em líquido pirolenhoso (\%); RCF: rendimento em carbono fixo (\%); TMV: teor de materiais voláteis (\%); TCz: teor de cinzas (\%); TCF: teor em carbono fixo (\%); PCSc: poder calorífico superior do

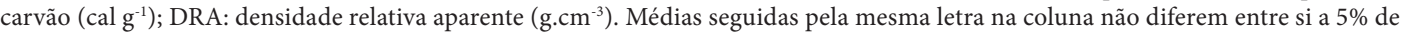
probabilidade pelo teste de Tukey.

uma dosagem de nitrogênio de 244,52 kg.ha ${ }^{-1}$ e o tratamento 1 de 5,72 kg.ha-1.

Os valores encontrados neste estudo estão em conformidade como os obtidos por Neves et al. (2011), Oliveira et al. (2010) e Botrel et al. (2007). Apesar da não diferença estatística, observa-se que o tratamento 3 foi o que apresentou o maior valor médio de rendimento em carbono fixo devido, provavelmente, ao maior teor de lignina na madeira.

\section{CONCLUSÃO}

A nutrição nitrogenada apresentou efeito significativo nas características de crescimento das árvores e estimativas de massa seca, tendo sido verificada tendência de aumento até a dosagem equivalente de $245,72 \mathrm{~kg} \cdot \mathrm{ha}^{-1}$. Entretanto, o mesmo efeito não foi observado na densidade básica da madeira.

As características químicas e energéticas da madeira não foram afetadas significativamente pela adubação nitrogenada, independentemente das fontes utilizadas.

Para o carvão vegetal, o efeito da dosagem de nitrogênio foi significativo somente para o rendimento em líquido pirolenhoso da carbonização, em que o menor rendimento ocorreu na ausência de nitrogênio (tratamento 1 ).

Aparentemente, o tratamento 3 foi o mais adequado para as características de crescimento da árvore, não interferindo no rendimento em carvão e em sua densidade relativa aparente.

\section{AGRADECIMENTOS}

Ao Conselho Nacional de Desenvolvimento Científico e Tecnológico (CNPq) e à APERAM Bioenergia

\section{STATUS DA SUBMISSÃO}

Recebido: 27 out., 2014

Aceito: 23 fev., 2017

\section{AUTOR(ES) PARA CORRESPONDÊNCIA}

\section{Claudinéia Olímpia Assis}

Departamento de Ciências Florestais - DCF, Universidade Federal de Lavras - UFLA, CP 3037, CEP 37200-000, Lavras, MG, Brasil e-mail: claudineia21@yahoo.com.br

\section{REFERENNCIAS}

Araújo EF, Gava JL, Souza AJ, Silveira RLVA. Crescimento de clones de Eucalyptus em resposta à aplicação de nitrogênio em espodossolo no sul da Bahia. In: Anais do Congresso Brasileiro de Ciência de Solo; 2003; Ribeirão Preto. Ribeirão Preto: Sociedade Brasileira de Ciência do Solo; 2003. CD-ROM.

Associação Brasileira de Normas Técnicas - ABNT. NBR 8633: carvão vegetal: determinação do poder calorífico. Rio de Janeiro: ABNT; 1984.

Associação Brasileira de Normas Técnicas - ABNT. NBR 11941: madeira: determinação da densidade básica. Rio de Janeiro: ABNT; 2003.

Associação Brasileira Técnica de Celulose e Papel - ABCTP. Celulose e papel. São Paulo: ABCTP; 1974. 6 p. 
Barbosa BM, Colodette JL, Cabral CPT, Gomes FJB, Silva VL. Efeito da fertilização na qualidade da madeira de Eucalyptus spp. Scentia Forestalis 2014; 42(101): 29-39.

Bilgen S, Kaygusuz K. The calculation of the chemical exergies of coal-based fuels by using the higher heating values. Applied Energy 2008; 85(8): 776-785. http://dx.doi. org/10.1016/j.apenergy.2008.02.001.

Botrel MCG, Trugilho PF, Rosado SCS, Silva JRM. Melhoramento genético das propriedades do carvão vegetal de Eucalyptus. Revista Árvore 2007; 31(3): 391-398. http://dx.doi.org/10.1590/S0100-67622007000300004.

Cooke EK, Martin A, Davis JM. Shortterm physiological and developmental responses to nitrogen availability in hybrid poplar. The New Phytologist 2005; 167(1): 41-52. PMid:15948828. http://dx.doi.org/10.1111/j.14698137.2005.01435.x.

Geßler A, Kopriva S, Rennenberg H. Regulation of nitrate uptake at the whole tree level: interaction between nitrogen compounds, cytokinins and carbon metabolism. Tree Physiology 2004; 24(12): 1313-1321. PMid:15465694. http://dx.doi.org/10.1093/treephys/24.12.1313.

Goldschimid O. Ultraviolet spectra. In: Sarkanen KV, Ludwig CH, editores. Lignins: occurrence, formation, structure and reations. New York: John Wiley \& Sons; 1971. p. 241-266.

Gomide JL, Demuner BJ. Determinação do teor de lignina em material lenhoso: método Klason modificado. O Papel 1986; 47(8): 36-38.

Huang C, Han L, Yang Z, Liu X. Ultimate analysis and heating value prediction of straw by near infrared spectroscopy. Waste Management 2009; 29(6): 1793-1797. PMid:19138837. http://dx.doi.org/10.1016/j.wasman.2008.11.027.

Jesus GL, Barros NF, Silva IR, Neves JCL, Henriques EP, Lima VC et al. Doses e fontes de nitrogênio na produtividade do eucalipto e nas frações da matéria orgânica em solo da região do cerrado de Minas Gerais. Revista Brasileira de Ciência do Solo 2012; 36(1): 201-214. http://dx.doi. org/10.1590/S0100-06832012000100021.

Kumar R, Pandey KK, Chandrashekar N, Mohan S. Effect of tree-age on calorific value and other fuel properties of Eucalyptus hybrid. Journal of Forest Research 2010; 21(4): 514-516. http://dx.doi.org/10.1007/s11676-010-0108-x.

MacDonald E, Hubert J. A review of the effects of silviculture on timber quality of Sitka Spruce. Forestry 2002; 75(2): 107-138. http://dx.doi.org/10.1093/forestry/75.2.107.
Neves TA, Protásio TP, Couto AM, Trugilho PF, Silva VO, Vieira CMM. Avaliação de clones de Eucalyptus em diferentes locais visando à produção de carvão vegetal. Pesquisa Florestal Brasileira 2011;31(68): 319-330. http:// dx.doi.org/10.4336/2011.pfb.31.68.319.

Oliveira AC, Carneiro ACO, Almeida W, Pereira BLC, Cardoso MT. Parâmetros de qualidade da madeira e do carvão vegetal de Eucalyptus pellita F. Muell. Scientia Forestalis 2010; 38(87): 431-439.

Oliveira AC, Rocha MFV, Pereira LCP, Carneiro ACO, Carvalho AMML, Vital BR. Avaliação de diferentes níveis de debastes nas propriedades da madeira e do carvão vegetal de Eucalyptus grandis $x$ Eucalyptus urophilla. Floresta 2012; 42(1): 59-68. http://dx.doi.org/10.5380/ rf.v42i1.26297.

Protásio TP, Bufalino L, Tonoli GHD, Couto AM, Trugilho PF. Guimarães Júnior, M. Relação entre o poder calorífico superior e os componentes elementares e minerais da biomassa vegetal. Pesquisa Florestal Brasileira, Colombo 2011; 31(66): 122-133. http://dx.doi.org/10.4336/2011. pfb.31.66.113.

Reis AA, Melo ICNA, Protásio TP, Trugilho PF, Carneiro ACO. Efeito de local e espaçamento na qualidade do carvão vegetal de um clone de Eucalyptus urophylla S.T. Blake. Floresta e Ambiente 2012; 19(4): 497-505. http:// dx.doi.org/10.4322/floram.2012.055.

Rigatto PA, Dedecek RA, Mattos JLM. Influência dos atributos do solo sobre a produtividade do Pinus taeda. Revista Árvore. Viçosa 2005; 29(5): 701-709.

Santana RS, Barros NF, Novais RF, Leite HG, Comerford NB. Alocação de nutrientes em plantios de eucalipto no Brasil. Revista Brasileira de Ciência do Solo 2008; 32(spe): 2723-2733. http://dx.doi.org/10.1590/S010006832008000700016.

Silva CR, Silveira RLVA, Camargo FRA, Patrocinio DD, Higashi EN. Crescimento de clone híbrido de Eucalyptus em função da aplicação de nitrogênio na presença e ausência de potássio. In: Anais do Congresso Brasileiro de Ciência do Solo; 2003; Ribeirão Preto. Ribeirão Preto: Sociedade Brasileira de Ciência do Solo; 2003. CD-ROM.

Smethurst P, Baillie C, Cherry M, Holz G. Fertilizer effects on lai and growth of four Eucalyptus nitens plantations. Forest Ecology and Management 2003; 176(1-3): 531-542. http://dx.doi.org/10.1016/S0378-1127(02)00226-8.

Taiz L, Zeiger E. Assimilação de nutrientes minerais. In: Taiz L, Zeiger E. Fisiologia vegetal. 4. ed. Porto Alegre: The Art of Medication; 2009. p. 317-330. 